\title{
An analysis of lectin agglutination as a means of sub-dividing gonococcal serovars
}

\author{
A. MOYES and H. YOUNG \\ STD Diagnostic Laboratory, Department of Medical Microbiology, University of Edinburgh Medical \\ School, Teviot Place, Edinburgh EH8 9AG
}

\begin{abstract}
Summary. Sixteen lectins were examined for their ability to agglutinate 298 strains of Neisseria gonorrhoeae. Seven lectins failed to agglutinate any of the strains; the remaining nine lectins gave 22 different agglutination patterns. The 298 strains were divided into 14 serovars with a single panel of monoclonal antibody typing reagents; lectin agglutination subdivided these into 57 serovar/lectin patterns. A combination of two monoclonal antibody serotyping panels divided the strains into 32 serovar combinations; lectin agglutination further subdivided these into 79 serovar/lectin patterns. There was no correlation between lectin pattern and serovar. Lectin agglutination is a simple supplementary typing method and could be particularly useful in micro-epidemiological studies.
\end{abstract}

\section{Introduction}

Lectins are natural proteins that are not part of immune mechanisms and that react with sugar residues. They have been used in the study of cell-surface carbohydrates of bacteria and can detect inter-strain variations in cell-wall carbohydrate composition ${ }^{1}$. The selectivity of lectins for microbial surfaces may be advantageous in epidemiological studies of bacterial disease.

The ability of animal and plant lectins to react with microbial substances has been known for some time. Summer and Howell ${ }^{2}$ first reported bacterial reactions with lectins by demonstrating the ability of Concanavalin A to agglutinate species of Mycobacterium and Actinomyces. In the late 1970s, agglutination of Neisseria gonorrhoeae by wheat germ lectin was advocated as a confirmatory test for identifying this organism. ${ }^{3}$ The interaction between lectins and members of the genus Neisseria has been well documented in several other studies and has been evaluated for identification ${ }^{4-7}$ and epidemiological typing. ${ }^{8,9}$ Vázquez and Berron ${ }^{10}$ examined lectin agglutination in relation to serogroup and auxotype. However, there have been no detailed studies correlating lectin agglutination with serotyping based on monoclonal antibodies (MAbs). ${ }^{11}$ In this study we examined the reactivity of 298 clinical isolates of $N$. gonorrhoeae with a panel of 16 lectins and assessed the increased epidemiological discrimination achieved by the combination of lectin agglutination and serotyping.

\section{Materials and methods}

\section{Bacterial strains}

A series of 298 clinical isolates of $N$. gonorrhoeae from patients attending the Genitourinary Medicine Unit, Edinburgh Royal Infirmary during 1988 and 1989 was studied. The isolates were identified as $N$. gonorrhoeae by the rapid carbohydrate utilisation test and the Phadebact Monoclonal GC test $:^{12} 149$ isolates were serogroup IA and 149 were serogroup IB. Serotyping of the isolates was performed as described previously ${ }^{13}$ with both the Genetic Systems (GS) panel of MAbs supplied by Dr C. Ison, St Mary's Hospital, London, and the Pharmacia (PH) panel of MAbs supplied by Dr S. Bygdeman, Karolinska Institute, Sweden. All of these MAbs are directed against epitopes on gonococcal outer-membrane protein I.

\section{Lectin agglutination}

Bacterial suspensions. Isolates were inoculated on to clear gonococcal (GC) agar and incubated at $37^{\circ} \mathrm{C}$ in an atmosphere of $\mathrm{CO}_{2} 5 \%$ in air for 18-24 h. Growth was harvested with a cotton swab into $1 \mathrm{ml}$ of phosphate-buffered saline, $\mathrm{pH} 7.2$ (PBS), to give a smooth suspension. The suspension was boiled for $10 \mathrm{~min}$, allowed to cool and adjusted to an optical density of 0.5 at $450 \mathrm{~nm}$ with a Titertek Multiskan MCC 340 spectrophotometer.

Lectins. Sixteen lectins were obtained from Sigma, reconstituted in distilled water to a concentration of $1 \mathrm{mg} / \mathrm{ml}$ and stored in small divided volumes at $-20^{\circ} \mathrm{C}$. For use, lectin solution was thawed and diluted with distilled water to the concentrations 
Table I. Panel of lectins and concentrations used

\begin{tabular}{lllc}
\hline Code & \multicolumn{1}{c}{ Lectin } & Common name & $\begin{array}{c}\text { Concentration } \\
(\mu \mathrm{g} / \mathrm{ml})\end{array}$ \\
\hline PNA & Arachis hypogea & Peanut & 62 \\
BSI & Bandeiraea simplicifolia I & Griffonia seeds & 62 \\
Cona & Concanavalin A & Jack bean & 250 \\
DBA & Dolichos biflorus & Horse gram & 62 \\
SBA & Glycine max & Soybean & 62 \\
HPA & Helix pomatia & Snail & 62 \\
SJA & Sophora japonica & Pagoda tree & 62 \\
LCH & Lens culinaris & Lentil & 62 \\
MPA & Maclura pomifera & Osage orange & 62 \\
PSA & Pisum sativum & Pea & 62 \\
STA & Solanium tuberosum & Potato & 62 \\
WGA & Triticum vulgaris & Wheat germ & 62 \\
UEA & Ulex europeaus & Gorse & 62 \\
PHA & Phaseolus vulgaris & Red kidney bean & 62 \\
BSII & Bandeiraea simplicifolia II & Griffonia seeds & 62 \\
LPA & Limulus polyphemus & Horseshoe crab & 62 \\
& & & \\
\hline
\end{tabular}

Table II. Lectin agglutination patterns of 298 strains of $N$. gonorrhoeae

\begin{tabular}{ccccccccccc}
\hline \multicolumn{7}{c}{ Agglutination with } & & & & Number \\
LNA & BSI & SBA & HPA & SJA & MPA & STA & WGA & BSII & of strains & patterns \\
\hline+ & + & + & + & + & + & + & + & - & 2 & LP12 \\
+ & + & + & + & + & - & + & + & - & 184 & LP1 \\
+ & + & + & + & + & - & + & + & + & 17 & LP3 \\
+ & + & + & + & + & - & - & + & - & 7 & LP6 \\
+ & + & + & + & - & - & + & + & - & 3 & LP9 \\
+ & + & + & - & + & - & + & + & - & 1 & LP15 \\
+ & + & + & - & - & - & - & + & - & 1 & LP16 \\
+ & - & + & - & - & - & - & - & - & 4 & LP8 \\
+ & - & + & + & + & - & + & + & - & 3 & LP10 \\
+ & - & + & + & + & - & - & + & - & 2 & LP13 \\
+ & - & + & + & - & - & - & + & - & 1 & LP17 \\
- & + & + & + & + & - & - & - & - & 3 & LP11 \\
- & + & + & + & + & - & + & + & - & 40 & LP2 \\
- & + & + & + & + & - & - & + & - & 10 & LP4 \\
- & + & + & + & - & - & + & + & - & 5 & LP7 \\
- & + & + & + & - & - & - & + & - & 2 & LP14 \\
- & + & + & - & + & - & + & + & - & 1 & LP18 \\
- & - & + & + & + & - & + & + & - & 8 & LP5 \\
- & - & + & + & + & - & - & + & - & 1 & LP19 \\
- & - & + & + & - & - & + & + & - & 1 & LP20 \\
- & - & + & + & - & - & - & + & - & 1 & LP21 \\
- & - & + & - & - & - & - & + & - & 1 & LP22 \\
\hline
\end{tabular}

described in previous studies. ${ }^{3-6,8,9}$ The lectins and concentrations used are shown in table I.

Agglutination tests. Standardised test suspension $(20 \mu \mathrm{l})$ was pipetted into two wells on a glass tile; $20 \mu \mathrm{l}$ of the appropriate lectin solution was added to one well and $20 \mu$ l of PBS was added to the other well. The suspensions were mixed and placed on a rotary shaker for $5 \mathrm{~min}$. Reactions were scored as positive when agglutination occurred in the lectin suspension but not in the control suspension. Strains showing agglutination in the control suspension were re-tested.

\section{Results}

Seven lectins did not agglutinate any of the 298 gonococcal isolates (ConA, DBA, LCH, PSA, UEA, PHA and LPA). The agglutination patterns with the remaining nine lectins are shown in table II. Twentytwo lectin patterns or groups were recognised: these are designated LP1-LP22 based on their frequency of occurrence. All isolates were agglutinated by soybean lectin (SBA). Only two isolates $(0.7 \%)$ were agglutinated by maclura pomifera lectin (MPA); four strains were agglutinated by PNA and SBA and three strains by BSI, SBA, HPA and SJA ; and seven isolates $(2 \cdot 3 \%)$ failed to agglutinate wheat germ lectin (WGA).

Combining lectin agglutination with serotyping with MAbs (table III) markedly increased the discrimination between isolates. The 298 isolates were divided into 14 different serovars with the GS panel of MAbs. 
Table III. Discrimination given by lectin agglutination and serotyping among 298 gonococcal isolates

\begin{tabular}{l|ccccc}
\hline & \multicolumn{5}{|c}{$\begin{array}{c}\text { Number of groups given by typing method(s) when } \\
\text { results are considered alone or in combination }\end{array}$} \\
\cline { 2 - 6 } Serogroup & Lectin & GS & Lectin/GS & GS/PH & GS/PH/Lectin \\
\hline IA isolates (149) & 13 & 4 & 22 & 4 & 22 \\
IB isolates (149) & 19 & 10 & 35 & 28 & 57 \\
Total & $22^{*}$ & 14 & 57 & 32 & 79 \\
\hline
\end{tabular}

GS, Genetic Systems MAbs. ${ }^{13}$

$\mathrm{PH}$, Pharmacia MAbs. ${ }^{13}$

* The same lectin pattern often occurred with isolates of serogroups IA and IB.

A combination of the GS panel with the PH MAb panel yielded 32 serovar combinations. A combination of GS panel serotype with lectin agglutination yielded 57 serovar/lectin patterns and a compilation of GS panel serotype with $\mathrm{PH}$ panel serotype and lectin agglutination yielded 79 serovar/lectin combinations.

The combination of serotyping and lectin agglutination enabled subdivision of serogroup IA and serogroup IB strains. The two most common lectin patterns (LP1 and LP2) accounted for $184(61.7 \%)$ and $40(13.4 \%)$ of strains, respectively (tables IV and V). The most common IA serovar was IA-2, representing $85.2 \%$ of the IA serotypes; this could be subdivided into 12 lectin patterns. Less discrimination was obtained with the remaining IA serovars: IA-4, IA-6 and IA-21 yielded two, five and three lectin patterns, respectively. The most common IB serovar was IB-2 (36.9\% of IB serotypes); unfortunately, the degree of further discrimination was less with IB-2 than with IA-2 serovars-only seven lectin patterns were recognised. Serovars IB-1 and IB-3 could be subdivided into 10 and six lectin patterns, respectively; the remaining seven IB serovars yielded only 12 serovar/lectin patterns.

\section{Discussion}

Typing of $N$. gonorrhoeae is useful in the control of gonococcal infection, e.g., in the recognition and control of micro-epidemics by detecting the occurrence of new strains or increased prevalence of existing ones. It is also useful in monitoring the spread of isolates with plasmid and chromosomally mediated antibiotic resistance and in recognising re-infections and double infections.

In this study, we have shown that agglutination with a panel of 16 animal and plant lectins subdivided 149 serogroup IA isolates into 13 types and 149 serogroup IB isolates into 19 types and merits consideration as a supplementary means for epidemiological typing of $N$. gonorrhoeae. A similar study with 101 strains of $N$. gonorrhoeae from outbreaks in Georgia, California and Hawaii, and a panel of 14 lectins, yielded 24 lectin patterns. ${ }^{8}$ Korting and Abeck ${ }^{14}$ distinguished 29
Table IV. Distribution of lectin patterns within serogroup IA isolates

\begin{tabular}{l|rrrrr}
\hline & \multicolumn{4}{|c}{ Number of isolates of serotype } & $\begin{array}{c}\text { Total } \\
\text { number of } \\
\text { Lectin pattern }\end{array}$ \\
\cline { 2 - 5 } & IA-2 & IA-4 & IA-6 & IA-21 & \\
\hline LP1 & 92 & 0 & 7 & 0 & 99 \\
LP2 & 13 & 4 & 4 & 0 & 21 \\
LP3 & 8 & 0 & 0 & 0 & 8 \\
LP4 & 1 & 0 & 1 & 0 & 2 \\
LP5 & 5 & 1 & 0 & 1 & 7 \\
LP6 & 1 & 0 & 1 & 0 & 2 \\
LP7 & 1 & 0 & 0 & 0 & 1 \\
LP8 & 0 & 0 & 0 & 0 & 0 \\
LP9 & 0 & 0 & 0 & 1 & 1 \\
LP10 & 1 & 0 & 1 & 0 & 2 \\
LP11 & 0 & 0 & 0 & 0 & 0 \\
LP12 & 2 & 0 & 0 & 0 & 2 \\
LP13 & 0 & 0 & 0 & 1 & 1 \\
LP14 & 1 & 0 & 0 & 0 & 1 \\
LP15 & 1 & 0 & 0 & 0 & 1 \\
LP16 & 0 & 0 & 0 & 0 & 0 \\
LP17 & 0 & 0 & 0 & 0 & 0 \\
LP18 & 0 & 0 & 0 & 0 & 0 \\
LP19 & 0 & 0 & 0 & 0 & 0 \\
LP20 & 1 & 0 & 0 & 0 & 1 \\
LP21 & 0 & 0 & 0 & 0 & 0 \\
LP22 & 0 & 0 & 0 & 0 & 0 \\
Total & 127 & 5 & 14 & 3 & 149 \\
& & & & & \\
\hline
\end{tabular}

lectin patterns amongst 102 gonococcal isolates from Munich with a panel of 14 lectins.

The four major lectin patterns reported here (LP1-LP4) accounted for $84 \%$ (251 out of 298) of isolates, which is similar to the results of serotyping studies in which a few major serovars account for the majority of strains. ${ }^{13,15-17}$

As seven of the lectins tested did not react with any of the strains, the panel could be reduced to nine lectins without any decrease in discrimination. Inclusion of ConA in any typing panel is debatable because it failed to agglutinate any of 54 strains, ${ }^{4}$ 193 strains, ${ }^{6}$ and 40 strains $^{8}$ tested in other studies. However, all 101 strains were agglutinated by ConA in the study by Korting and Abeck, ${ }^{14} 61.8 \%$ of 140 strains tested by Vazquez and Berron were agglutinated ${ }^{10}$ and two of 101 penicillinase-producing and non-penicillinase-producing strains examined in a 
Table V. Distribution of lectin patterns within serogroup IB isolates

\begin{tabular}{|c|c|c|c|c|c|c|c|c|c|c|c|}
\hline \multirow{2}{*}{$\begin{array}{l}\text { Lectin } \\
\text { pattern }\end{array}$} & \multicolumn{10}{|c|}{ Number of isolates of serotype } & \multirow{2}{*}{$\begin{array}{c}\text { Total } \\
\text { number of } \\
\text { isolates }\end{array}$} \\
\hline & IB-1 & IB-2 & IB-3 & IB-4 & IB-6 & IB-7 & IB-8 & IB-16 & IB-17 & IB-29 & \\
\hline LP1 & 19 & 37 & 17 & 2 & 8 & 0 & 0 & 2 & 0 & 0 & 85 \\
\hline LP2 & 5 & 5 & 8 & 0 & 0 & 1 & 0 & 0 & 0 & 0 & 19 \\
\hline LP3 & 0 & 8 & 0 & 0 & 0 & 0 & 0 & 0 & 0 & 1 & 9 \\
\hline LP4 & 3 & 1 & 2 & 0 & 1 & 1 & 0 & 0 & 0 & 0 & 8 \\
\hline LP5 & 0 & 2 & 0 & 0 & 0 & 0 & 0 & 0 & 0 & 0 & 2 \\
\hline LP6 & 4 & 1 & 0 & 0 & 0 & 0 & 0 & 0 & 0 & 0 & 5 \\
\hline LP7 & 0 & 0 & 1 & 2 & 0 & 0 & 0 & 0 & 0 & 0 & 3 \\
\hline LP8 & 4 & 0 & 0 & 0 & 0 & 0 & 0 & 0 & 0 & 0 & 4 \\
\hline LP9 & 1 & 0 & 0 & 0 & 0 & 1 & 0 & 0 & 0 & 0 & 2 \\
\hline LP10 & 1 & 0 & 0 & 0 & 0 & 0 & 0 & 0 & 0 & 0 & 1 \\
\hline LP11 & 3 & 0 & 0 & 0 & 0 & 0 & 0 & 0 & 0 & 0 & 3 \\
\hline LP12 & 0 & 0 & 0 & 0 & 0 & 0 & 0 & 0 & 0 & 0 & 0 \\
\hline LP13 & 0 & 0 & 0 & 0 & 1 & 0 & 0 & 0 & 0 & 0 & 1 \\
\hline LP14 & 0 & 0 & 1 & 0 & 0 & 0 & 0 & 0 & 0 & 0 & 1 \\
\hline LP15 & 0 & 0 & 0 & 0 & 0 & 0 & 0 & 0 & 0 & 0 & 0 \\
\hline LP16 & 1 & 0 & 0 & 0 & 0 & 0 & 0 & 0 & 0 & 0 & 1 \\
\hline LPI7 & 1 & 0 & 0 & 0 & 0 & 0 & 0 & 0 & 0 & 0 & 1 \\
\hline LP18 & 0 & 0 & 1 & 0 & 0 & 0 & 0 & 0 & 0 & 0 & 1 \\
\hline LP19 & 0 & 1 & 0 & 0 & 0 & 0 & 0 & 0 & 0 & 0 & 1 \\
\hline LP20 & 0 & 0 & 0 & 0 & 0 & 0 & 0 & 0 & 0 & 0 & 0 \\
\hline LP21 & 0 & 0 & 0 & 0 & 0 & 0 & 0 & 0 & 1 & 0 & 1 \\
\hline LP22 & 0 & 0 & 0 & 0 & 0 & 0 & 1 & 0 & 0 & 0 & 1 \\
\hline Total & 42 & 55 & 30 & 4 & 10 & 3 & 1 & 2 & 1 & 1 & 149 \\
\hline
\end{tabular}

further study by Schalla et al. ${ }^{9}$ The reasons for the variations in results in these studies is unclear, but it is unlikely to be due to the inclusion of minor serovars or types because of the large number of strains tested. Similarly, MPA might also be omitted from the panel because only two of the 298 isolates were reactive and none were reactive in two further studies with a total of 247 strains. ${ }^{4,6}$

All the strains in this study gave a positive reaction with SBA, which is one of the criteria in the five tests comprising lectins and chromogenic substrates ${ }^{18}$ for the rapid identification of strains of $N$. gonorrhoeae. SBA agglutination in this case was useful in the identification of strains that were not agglutinated by WGA.

We found that seven $(2 \cdot 3 \%)$ strains failed to agglutinate WGA compared with $0-5.9 \%$ of strains in several other studies. ${ }^{5,6,8-10,18}$ WGA agglutination cannot, therefore, be relied upon as a single criterion for the confirmatory identification of $N$. gonorrhoeae ${ }^{3}$ All seven WGA-negative strains belonged to serovar IB-1/Bropyt; a further 16 isolates of serovar IB-1 Bropyt gave a positive reaction with WGA and could be differentiated into six lectin patterns.

A combination of lectin pattern with serotype markedly increased the discrimination over that obtained with either method alone. The greatest overall discrimination was given by lectin agglutination in combination with both the GS and PH serotyping panels of MAbs; this resulted in 79 serovar/lectin combinations. We have shown that the major serovars, such as IA-2 and IB-2, can be subdivided into 12 and seven lectin patterns, respectively. This is relevant in the micro-epidemiological approach to gonorrhoea control, in which auxotyping has been the primary means of further subdivision of serovars, i.e., auxotype/serovar classification. ${ }^{19}$ In this study we have been unable to show any correlation between lectin pattern and serovar when either a single panel or a combination of panels of MAbs was used. Others have been unable to show any correlation between lectin pattern and auxotype ${ }^{8,14}$ or between lectin pattern and serovar. ${ }^{8}$

We have shown previously that the use of two panels of MAbs (GS and PH panels) increases discrimination in typing $N$. gonorrhoeae ${ }^{13}$ Provided that lectin agglutination is shown to be reproducible, the combination of GS/PH serovar with lectin pattern could provide sufficient discrimination to be a simple alternative to auxotype/serovar classification. There is, however, a need for an agreed reference panel of lectins to be used in such an epidemiological typing system.

\section{References}

1. Pistole TG. Interaction of bacteria and fungi with lectins and lectin-like substances. Annu Rev Microbiol 1981; 35: $85-112$.

2. Summer JB, Howell SF. The identification of the hemag-

glutination of the jack bean with concanavalin A. $J$ Bacteriol 1936; 32: 227-237.

3. Schaefer RL, Keller KF, Doyle RJ. Lectins in diagnostic microbiology: use of wheat germ agglutinin for laboratory identification of Neisseria gonorrhoeae. J Clin Microbiol $1979 ; 10: 669-672$. 
4. Allen PZ, Connelly MC, Apicella MA. Interaction of lectins with Neisseria gonorrhoeae. Can J Microbiol 1980; 26: 468-474.

5. Curtis GDW, Slack MPE. Wheat-germ agglutination of Neisseria gonorrhoeae. Br J Vener Dis 1981; 57: 253-255.

6. Doyle RJ. Nedjat-Haeim F, Keller KF, Frasch CE. Diagnostic value of interactions between members of the family Neisseriaceae and lectins. J Clin Microbiol 1984; 19: 383-387.

7. Frasch CE. Role of lipopolysaccharide in wheat germ agglutinin-mediated agglutination of Neisseria meningitidis and Neisseria gonorrhoeae. J Clin Microbiol 1980; 12: 498-501.

8. Schalla WO, Rice RJ, Biddle JW, Jeanlouis Y, Larsen SA, Whittington WL. Lectin characterization of gonococci from an outbreak caused by penicillin-resistant Neisseria gonorrhoeae. J Clin Microbiol 1985; 22 : 481-483.

9. Schalla WO, Whittington WL, Rice RJ, Larsen SA. Epidemiological characterization of Neisseria gonorrhoeae by lectins. J Clin Microbiol 1985; 22: 379-382.

10. Vázquez JA, Berron S. Lectin agglutination test as an epidemiological marker for Neisseria gonorrhoeae. Genitourin Med 1990; 66: 302

11. Sarafian SK, Knapp JS. Molecular epidemiology of gonorrhoea. J Clin Microbiol 1989; 2 Suppl: S49-S55.

12. Young H, Moyes A. Utility of monoclonal antibody coagglutination to identify Neisseria gonorrhoeae. Genitourin Med 1989; 65: 8-13.

13. Moyes A, Young H. Epidemiological typing of Neisseria gonorrhoeae: a comparative analysis of three monoclonal antibody serotyping panels. Eur J Epidemiol 1991; 7 : 311-319.

14. Korting HC, Abeck D. Lectin-typing-a powerful epidemiological marker system for Neisseria gonorrhoeae infections. Zentralbl Bakteriol Mikrobiol Hyg $(A)$ 1988; 269 : 506-512.

15. Bygdeman SM. Polyclonal and monoclonal antibodies applied to the epidemiology of gonococcal infection. In : Young $\mathrm{H}$ McMillan A (eds) Immunological diagnosis of sexually transmitted diseases. (Clinical and biochemical analysis 23) New York, Marcel Dekker. 1987: 117

16. Knapp JS, Tam MR, Nowinski RC, Holmes KK, Sandström EG. Serological classification of Neisseria gonorrhoeae with use of monoclonal antibodies to gonococcal outer membrane protein I. J Infect Dis 1984; 150: 44-48.

17. Young H, Moyes A, Robertson DHH et al. Gonococcal infection within Scotland: antigen heterogeneity and antibiotic susceptibility to infecting strains. Eur $J$ Epidemiol $1990 ; 6: 1-8$.

18. Yajko DM, Chu A, Hadley WK. Rapid confirmatory identification of Neisseria gonorrhoeae with lectins and chromogenic substrates. J Clin Microbiol 1984; 19: 380-382.

19. Knapp JS, Sandström EG, Holmes KK. Overview of epidemiological and clinical applications of auxotype/serovar classification of Neisseria gonorrhoeae. In: Schoolnik GK, Brooks GF, Falkow S et al. (eds) The pathogenic neisseria. Washington DC, American Society for Microbiology. 1985: 6-12. 